\title{
LETTERS-CONCISE RESEARCH REPORTS \\ Monitoring Health Effects of Vaping Discussed on Twitter in 2018 and 2019
}

$\mathrm{J}$ Gen Intern Med 37(3):673-5

DOI: $10.1007 / \mathrm{s} 11606-021-06705-9$

(c) The Author(s) 2021

\section{INTRODUCTION}

The USA is currently witnessing a vaping epidemic. Despite the rising popularity of electronic nicotine delivery systems (ENDS) or e-cigarette use (vaping), there is a limited understanding of public perceptions about the health effects of these products. While scientific evidence about the long-term health effects is inconclusive, emerging findings suggest that vaping is linked to cancer, cardiovascular, respiratory, and other health issues. ${ }^{1}$ An understanding of public perceptions about the health effects of vaping can help identify priority areas for patient education in clinician settings. Social media surveillance of public perceptions of the health effects of vaping may inform patient-provider interactions, and complement findings from traditional survey research at a low cost. ${ }^{2}$ This study characterizes public conversations about the health effects of vaping on Twitter during 2018 and 2019.

\section{METHODS}

Vaping-related posts $(n=2,516,664$ posts in 2018, $n=$ $2,731,399$ posts in 2019 , total $n=5,248,063$ ) were obtained from January 1, 2018, to December 31, 2019, using Twitter's Streaming Application Program Interface (API). Data cleaning procedures included removal of retweets so that each observation could be treated as an independent unit of observation, and removal of posts from bot accounts. ${ }^{3}$ Posts containing health-related references to e-cigarettes were identified using two dictionaries: (a) the Unified Medical Language System ${ }^{\circledR}$ (UMLS) Consumer Health Vocabulary (CHV), ${ }^{4}$ comprising $n=13,479$ informal, common medical terms used by consumers and health care professionals; and (b) a list of $n=177$ additional informal terms corresponding to the CHV terms, wherever relevant (e.g., informal term of "inebriation" is "drunk"). Keywords from the two dictionaries encompassed health diseases and symptoms (referred to as "health effects" in this study). A total sample of $n=667,140(n=257,620$ posts in 2018; $n=409,520$ posts in 2019) e-cigarette-related

Received October 22, 2020

Accepted March 8, 2021

Published online April 9, 2021 posts with mentions of health effects were identified. Each post in the analytic sample was then classified to one or more of the 14 categories of health effects (see Table 1).

\section{RESULTS}

Percentage distribution of categories of health effects by 2018 and 2019 is reported in Figure 1. In 2018, Neurological effects ( $n=44,345 ; 17.2 \%$ ) were the most common, followed by Mental Health $(n=31,227 ; 12.1 \%)$ and Death $(n=27,040$; $10.5 \%)$. In 2019, Death was most commonly referenced ( $n=$ $77,719 ; 19.0 \%)$, followed by references to Neurological $(n=$ $67,504 ; 16.5 \%)$ and Respiratory $(n=48,875 ; 11.9 \%)$ health effects in 2019. Compared to 2018, Death, Mental health, and Respiratory health effects were more prevalent in 2019. Respiratory health effects emerged as part of the top five categories in 2019.

\section{DISCUSSION}

Neurological, Death, Mental Health, and Respiratory were all predominant categories of vaping-related health effects during 2018 and 2019 on Twitter. These findings may be relevant to primary healthcare providers as they counsel patients on the health risks associated with e-cigarette use. ${ }^{5}$ Armed with the insights from this study, clinicians can help patients better understand ways in which vaping may contribute to the abovementioned health effects. Chronic health conditions such as Cancer, cardiovascular diseases may also warrant consideration while discussing the potential long-term health

Table 1 Predominant Categories of Health Effects and Example Keywords

\begin{tabular}{ll}
\hline \hline Health categories & Example keywords \\
\hline Neurological & Coma, dizzy, lightheaded \\
Mental Health & PTSD, ADHD, jittery \\
Death & Die, kill, lost life \\
Injury & Injury, rupture, wound, bruise \\
Respiratory & Cough, wheeze, black lung \\
Pain & Painful, achy, cramping \\
Cancer & Cancer, tumor, malignant \\
Gastrointestinal & Belly, belch, vomit, puke \\
Cardiovascular & Stroke, heart attack, blood pressure \\
Weight-related & Fat, obese, weight, stoutness \\
Stress & Stressed, cortisol \\
Immunity & Flu, common cold, allergy \\
Pregnancy/In-utero & Pregnant, preggers, miscarriage \\
Other & Anemia, jaundice, mumps \\
\hline
\end{tabular}




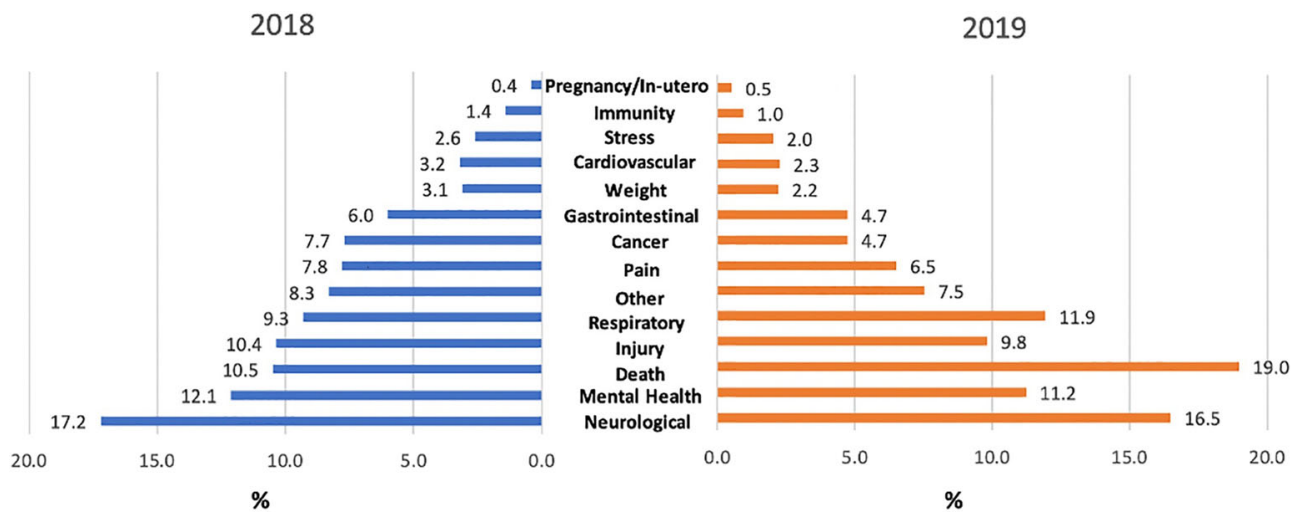

Figure 1 Percentage distribution of categories of health effects during 2018 and 2019.

risks of vaping since they may be less salient among patients. Respiratory health effects, a predominant category in 2019, may be potentially attributable to the outbreak of lung injuries associated with the use of vape products during that year. ${ }^{6}$ Findings will also inform future survey-based public health surveillance efforts and toxicology research examining longterm and short-term health effects of vaping.

This study drew data from public Twitter posts. Findings may not generalize to other social media platforms and may not represent data from individuals with private Twitter accounts. Specific references to the biological basis of the health effects (e.g., mentions of blocked arteries in the context of heart dysfunction) may not be captured in this study. While this study collected data from two different years, findings may not extend to other time periods.

Despite these limitations, findings may serve as a useful baseline to identify emerging categories and monitor predominant categories of perceived health effects of vaping in general and by source (e.g., news organizations, public health agencies) in the future. Unexpected peaks in reported categories of health effects may also help inform the surveillance of potential vaping-related outbreaks. Taken altogether, characterizing the public's expressions of the health effects of vaping through Twitter data can offer insights to medical providers and inform survey-based research in the future.

Acknowledgements: The authors would like to thank Dr. Pablo Barberá, Associate Professor, Department of Political Science and International Relations at the University of Southern California, for his valuable inputs.

Anuja Majmundar, $P h D, M B A, M A^{1}$

Jon-Patrick Allem, $\mathrm{PhD}^{2}$

Tess Boley Cruz, $\mathrm{Ph} \mathrm{D}^{2}$

Jennifer B. Unger, $P h D^{2}$

Mary Ann Pentz, $\mathrm{PhD}^{2}$

${ }^{1}$ Department of Preventive Medicine, Keck School of Medicine, University of Southern California,

Los Angeles, CA, USA

${ }^{2}$ American Cancer Society,

, DCWashington, DC, USA
Corresponding Author: Anuja Majmundar, PhD, MBA, MA; Department of Preventive Medicine, Keck School of Medicine, University of Southern California, Los Angeles, CA, USA (e-mail: anuja.majmundar@cancer.org).

Author Contribution Conceptualization: AM

Data collection: $A M$

Data analysis: $A M$

First draft of the manuscript: $A M$

Critical review of the manuscript: AM, JPA, TBC, JBU, MAP

Funding Research reported in this publication was supported by Grant \# U54CA180905 from the National Cancer Institute and the FDA Center for Tobacco Products (CTP).

\section{Declarations:}

The content is solely the responsibility of the authors and does not necessarily represent the official views of the NIH or FDA. The first author was affiliated with the University of Southern California, Department of Preventive Medicine during data colelction, analysis and manuscript preparation.

Conflict of Interest: The authors declare that they do not have a conflict of interest.

Open Access This article is licensed under a Creative Commons Attribution 4.0 International License, which permits use, sharing, adaptation, distribution and reproduction in any medium or format, as long as you give appropriate credit to the original author(s) and the source, provide a link to the Creative Commons licence, and indicate if changes were made. The images or other third party material in this article are included in the article's Creative Commons licence, unless indicated otherwise in a credit line to the material. If material is not included in the article's Creative Commons licence and your intended use is not permitted by statutory regulation or exceeds the permitted use, you will need to obtain permission directly from the copyright holder. To view a copy of this licence, visit http://creativecommons. org/licenses/by/4.0/.

\section{REFERENCES}

1. Glantz SA, Bareham DW. E-Cigarettes: Use, Effects on Smoking, Risks, and Policy Implications. Annu Rev Public Health. 2018;39(1):215-235.

2. Allem J-P, Ferrara E, Uppu SP, Cruz TB, Unger JB. E-cigarette surveillance with social media data: Social bots, emerging topics, and trends. JMIR Public Health Surveillance. 2017;3(4):e98.

3. Allem J-P, Dharmapuri L, Unger JB, Cruz TB. Characterizing JUULrelated posts on Twitter. Drug Alcohol Depend. 2018;190:1-5. 
4. Zeng QT, Tse T. Exploring and developing consumer health vocabularies. Journal of American Medical Informatics Association. 2006; 13(1):24-29.

5. Peterson EB, Fisher CL, Zhao X. Pediatric primary healthcare providers' preferences, experiences and perceived barriers to discussing electronic cigarettes with adolescent patients. Journal of Communication in Healthcare. 2018; 11(4):245-251.
6. CDC. Outbreak of Lung Injury Associated with the Use of E-Cigarette, or Vaping, Products. https://www.cdc.gov/tobacco/basic_information/e-cigarettes/severe-lung-disease.html. Published 2020. Accessed 29 Jan 2020

Publisher's Note: Springer Nature remains neutral with regard to jurisdictional claims in published maps and institutional affiliations. 